\title{
Rigidity of fiber-preserving quasisymmetric maps
}

\author{
Enrico Le Donne and Xiangdong Xie
}

January 7, 2015

\begin{abstract}
We show that fiber-preserving quasisymmetric maps are biLipschitz. As an application, we show that quasisymmetric maps on Carnot groups with reducible first stratum are biLipschitz.
\end{abstract}

Keywords. quasisymmetric map, rigidity, Carnot group, reducible first stratum. Mathematics Subject Classification (2010). 30L10, 22E25, 53C17.

\section{Introduction}

In this paper we study the rigidity property of quasisymmetric maps that preserve a foliation. We show that, under quite general conditions, such quasisymmetric maps are biLipschitz. We then give an application to quasisymmetric maps between Carnot groups.

Quasisymmetric maps that preserve a foliation arise when one studies the rigidity property of quasiisometries between negatively curved solvable Lie groups, see [Pi], [SX], [X1], X4. It is well known that a negatively curved space has an ideal boundary, and quasiisometries between negatively curved spaces correspond to quasisymmetric maps between the ideal boundaries. Rigidity properties of the quasiisometries correspond to the rigidity properties of the quasisymmetric maps. The ideal boundary of a negatively curved solvable Lie group is a nilpotent Lie group equipped with a homogeneous distance. Recent results suggest that very often the quasisymmetric maps on such nilpotent Lie groups must be biLipschitz [P], [CO], [CR], [SX], [X1], [X2], [X4], [X5].

There are usually two steps in the proof of the above mentioned rigidity property of the quasisymmetric maps. The first step is to show that the quasisymmetric map preserves a certain foliation. The second step is to show that a quasisymmetric map must be biLipschitz if it preserves a foliation. The main purpose of this paper is to take a closer look at the second step in the context of quasisymmetric maps between general metric spaces. We hope that this will be useful in the eventual (hopefully) complete solution of the rigidity of quasiisometries between negatively curved solvable Lie groups.

One often has to deal with quasimetrics instead of metrics while studying the ideal boundary of negatively curved spaces. For this reason we state our main result for quasimetric spaces. Recall that a function $d: X \times X \rightarrow[0, \infty)$ is a quasimetric on a set $X$ if 
(1) $d$ is symmetric, that is, $d\left(x_{1}, x_{2}\right)=d\left(x_{2}, x_{1}\right)$ for all $x_{1}, x_{2} \in X$;

(2) $d\left(x_{1}, x_{2}\right)=0$ if and only if $x_{1}=x_{2}$;

(3) there exists some constant $M \geq 1$ such that $d\left(x_{1}, x_{3}\right) \leq M \cdot\left(d\left(x_{1}, x_{2}\right)+d\left(x_{2}, x_{3}\right)\right)$ for all $x_{1}, x_{2}, x_{3} \in X$.

We call $M$ a quasimetric constant of $d$. A subset $A$ of a quasimetric space $X$ is called closed if for any $x \in X$ and any sequence $\left\{a_{j}\right\} \subset A$, the condition $d\left(a_{j}, x\right) \rightarrow 0$ implies $x \in A$.

To state our main result, we introduce the notion of "fibered quasimetric space". To do that we first specify the concept of parallelism for sets. We say that two closed subsets $U$ and $V$ of a quasimetric space $X$ are parallel if there is a constant $a>0$ such that $d(u, V)=$ $d(v, U)=a$ for every $u \in U$ and every $v \in V$. Recall that $d(u, V):=\inf \{d(u, v): v \in V\}$. It is easy to check that in this case we have $d(U, V)=H D(U, V)=a$, where

$$
d(U, V):=\inf \{d(u, v) \mid u \in U, v \in V\},
$$

and $H D(U, V)$ denotes the Hausdorff distance between $U$ and $V$ :

$$
H D(U, V):=\sup (\{d(u, V): u \in U\} \cup\{d(v, U): v \in V\}) .
$$

The following definition provides the setting for our main theorem.

Definition 1.1 (Fibered quasimetric space). Let $\alpha>0, L \geq 1$. We say that a quasimetric space $X$ is an $(\alpha, L)$-fibered quasimetric space if $X$ admits a cover $\mathcal{U}$ by closed pairwise disjoint subsets, called fibers, with the following properties:

Fibers are snow-flake equivalent to unbounded geodesic spaces:

for each $U \in \mathcal{U}$, there exists an unbounded geodesic space $(\widetilde{U}, d)$ such that $U$ is $L$-biLipschitz to $\left(\widetilde{U}, d^{\alpha}\right)$

Parallel fibers are not isolated:

for any $U \in \mathcal{U}$, there exists a sequence $U_{i} \in \mathcal{U}$ such that $U_{i}$ and $U$ are distinct, parallel, and $H D\left(U_{i}, U\right) \rightarrow 0$ as $i \rightarrow \infty$;

Fibers have positive distance:

for any two distinct $U, V \in \mathcal{U}, d(U, V)>0$;

Non-parallel fibers diverge:

if $U, V \in \mathcal{U}$ are not parallel, then $H D(U, V)=\infty$.

Given $K \geq 1$ and $C>0$, we say that a bijection $F: X \rightarrow Y$ between two quasimetric spaces is a $(K, C)$-quasi-similarity if

$$
\frac{C}{K} \cdot d\left(x_{1}, x_{2}\right) \leq d\left(F\left(x_{1}\right), F\left(x_{2}\right)\right) \leq C K \cdot d\left(x_{1}, x_{2}\right), \quad \forall x_{1}, x_{2} \in X .
$$

Clearly a map is a quasi-similarity if and only if it is biLipschitz. The point here is that often there is control on $K$ but not on $C$. In this case, the notion of quasi-similarity provides more information about the distortion. 
The following is our main result and states that any fiber-preserving quasisymmetric map between fibered quasimetric spaces is a quasi-similarity (and hence biLipschitz). See Section 2.1 for the definition of quasisymmetric map.

Theorem 1.1. Let $X, Y$ be $(\alpha, L)$-fibered quasimetric spaces for some $\alpha>0$ and $L \geq 1$. Suppose $F: X \rightarrow Y$ is an $\eta$-quasisymmetric map that sends fibers of $X$ homeomorphically onto fibers of $Y$. Then $F$ is a $(K, C)$-quasi-similarity, where $K$ depends only on $\eta, \alpha, L$ and the quasimetric constants of $X, Y$.

We remark that for the validity of Theorem 1.1 the condition $d(u, V)=d(v, U)=a$ for parallel fibers can not be replaced by the weaker condition $H D(U, V)<\infty$. See the end of Section 3 for an example.

Theorem 1.1 in particular implies that all quasiconformal maps of the subRiemannian Heisenberg groups that send vertical lines to vertical lines must be biLipschitz, see Proposition 4.5. See [T] and [BHT] for the construction of such maps. We remark that there exist biLipschitz maps of Heisenberg groups that send vertical lines to curves that are not vertical lines, see [X3].

Our main application of Theorem 1.1 is to quasisymmetric maps on Carnot groups with reducible first stratum.

Let $G$ be a Carnot group. See Section 2.2 for a brief introduction to Carnot groups. Let $V_{1} \oplus \cdots \oplus V_{r}$ be the stratification of the Lie algebra $\mathfrak{g}$ of $G$. For each $t>0$, the standard dilation $\lambda_{t}: \mathfrak{g} \rightarrow \mathfrak{g}$ is defined by $\lambda_{t}(v)=t^{i} v$ for $v \in V_{i}$. A Lie algebra isomorphism $A: \mathfrak{g} \rightarrow \mathfrak{g}$ is called a strata-preserving automorphism if $A\left(V_{i}\right)=V_{i}$, for $i=1, \ldots, r$. Let $\operatorname{Aut}_{*}(\mathfrak{g})$ be the group of strata-preserving automorphisms of $\mathfrak{g}$. We say $V_{1}$ is reducible (or the first stratum of $\mathfrak{g}$ is reducible) if there is a non-trivial proper linear subspace $W_{1} \subset V_{1}$ such that $A\left(W_{1}\right)=W_{1}$ for every $A \in \operatorname{Aut}_{*}(\mathfrak{g})$.

The main consequence of Theorem 1.1 is the following theorem. Recall that a Carnot group is a metric space equipped with a particular Carnot-Carathéodory distance, see Section 2.2 ,

Theorem 1.2. Let $G$ be a Carnot group with reducible first stratum. Then every quasisymmetric map $F: G \rightarrow G$ is a quasi-similarity, quantitatively.

Theorem 1.2 is quantitatively in the sense that for any Carnot group $G$ and for any $\eta \in \operatorname{Homeo}([0, \infty))$ there exists a constant $K$ depending only on $G$ and $\eta$ such that every $\eta$-quasisymmetric map $F: G \rightarrow G$ is a $(K, C)$-quasi-similarity for some $C$.

We remark that in [CO], Cowling and Ottazzi proved that all smooth quasiconformal maps on rigid Carnot groups are affine maps (an affine map is the composition of a left translation and a strata-preserving automorphism) and so in particular are biLipschitz. On the other hand, it is shown in [X5] that if $N$ is a non-rigid Carnot group other than the Euclidean groups and some very particular type of product groups, then every quasisymmetric map of $N$ is biLipschitz. We note that Carnot groups with reducible first stratum include both rigid and non-rigid groups. In Theorem 1.2 there is no assumption on the regularity of the quasisymmetric map.

In Section 2 we recall some basic definitions and facts that are used later. In Section 3 we prove Theorem 1.1. In Section 4 we prove Theorem 1.2 and also give two other applications 
of Theorem 1.1; quasiconformal maps of Heisenberg groups that send vertical lines to vertical lines, and a new proof of a theorem of Dymarz [D] on quasisymmetric maps of ideal boundary of certain amenable hyperbolic locally compact groups.

Acknowledgment. This work was initiated when the second-named author visited the University of Jyvaskyla. He would like to thank University of Jyvaskyla for financial support and excellent working conditions. He also acknowledges support from NSF grant DMS1265735 and Simons Foundation grant \#315130.

\section{Preliminaries}

In this section we collect definitions and results needed later. We first recall the definitions of quasiconformal and quasisymmetric maps (Section 2.1). Then we review the basic definitions related to Carnot groups in Section 2.2, the BCH formula in Section 2.3 and Pansu Differentiability Theorem in Section 2.4.

\subsection{Quasiconformal and quasisymmetric maps}

A map $F: X \rightarrow Y$ between two quasimetric spaces is continuous if for any $x \in X$ and any sequence $\left\{x_{j}\right\} \subset X$, the condition $\lim _{j \rightarrow \infty} d\left(x, x_{j}\right)=0$ implies $\lim _{j \rightarrow \infty} d\left(F(x), F\left(x_{j}\right)\right)=0$.

Let $F: X \rightarrow Y$ be a bijection between two quasimetric spaces. For $x \in X$ and $t>0$, define

$$
H_{F}(x, t)=\frac{\sup \left\{d\left(F\left(x^{\prime}\right), F(x)\right) \mid d\left(x^{\prime}, x\right) \leq t\right\}}{\inf \left\{d\left(F\left(x^{\prime}\right), F(x)\right) \mid d\left(x^{\prime}, x\right) \geq t\right\}} .
$$

The map $F$ is called $\lambda$-quasiconformal if both $F$ and $F^{-1}$ are continuous and

$$
\limsup _{t \rightarrow 0} H_{F}(x, t) \leq \lambda
$$

for all $x \in X$. We say $F$ is quasiconformal if it is $\lambda$-quasiconformal for some $\lambda \geq 1$.

Let $\eta:[0, \infty) \rightarrow[0, \infty)$ be a homeomorphism. A bijection $F: X \rightarrow Y$ between two quasimetric spaces is $\eta$-quasisymmetric if both $F$ and $F^{-1}$ are continuous and for all distinct triples $x, y, z \in X$, we have

$$
\frac{d(F(x), F(y))}{d(F(x), F(z))} \leq \eta\left(\frac{d(x, y)}{d(x, z)}\right)
$$

If $F: X \rightarrow Y$ is an $\eta$-quasisymmetry, then $F^{-1}: Y \rightarrow X$ is an $\eta_{1}$-quasisymmetry, where $\eta_{1}(t)=\left(\eta^{-1}\left(t^{-1}\right)\right)^{-1}$, see $[\mathrm{V}$, Theorem 6.3]. A bijection between quasimetric spaces is said to be quasisymmetric if it is $\eta$-quasisymmetric for some $\eta$.

We remark that quasisymmetric maps between general quasimetric spaces are quasiconformal. In the case of Carnot groups, and more generally Loewner spaces, a quasiconformal homeomorphism is locally quasisymmetric, see [HK, Theorem 4.7]. 


\subsection{Stratified Lie algebras and Carnot groups}

A stratified Lie algebra is a finite-dimensional Lie algebra $\mathfrak{g}$ over $\mathbb{R}$ together with a direct sum decomposition $\mathfrak{g}=V_{1} \oplus V_{2} \oplus \cdots \oplus V_{s}$ of nontrivial vector subspaces such that $\left[V_{1}, V_{i}\right]=V_{i+1}$ for all $1 \leq i \leq s$, where we set $V_{s+1}=\{0\}$. The integer $s$ is called step or degree of nilpotency of $\mathfrak{g}$. Every stratified Lie algebra $\mathfrak{g}=V_{1} \oplus V_{2} \oplus \cdots \oplus V_{s}$ admits a one-parameter family of automorphisms $\lambda_{t}: \mathfrak{g} \rightarrow \mathfrak{g}, t \in(0, \infty)$, where $\lambda_{t}(x)=t^{i} x$ for $x \in V_{i}$.

Let $\mathfrak{g}=V_{1} \oplus V_{2} \oplus \cdots \oplus V_{s}$ and $\mathfrak{g}^{\prime}=V_{1}^{\prime} \oplus V_{2}^{\prime} \oplus \cdots \oplus V_{s}^{\prime}$ be two stratified Lie algebras. A Lie algebra isomorphism $\phi: \mathfrak{g} \rightarrow \mathfrak{g}^{\prime}$ is such that $\phi\left(V_{i}\right)=V_{i}^{\prime}$ for all $1 \leq i \leq s$ if and only if it commutes with $\lambda_{t}$ for all $t>0$; that is, if $\phi \circ \lambda_{t}=\lambda_{t} \circ \phi$. We call such isomorphisms strata preserving.

Let $G$ be a connected, simply connected Lie group whose Lie algebra is stratified as $\operatorname{Lie}(G)=V_{1} \oplus \cdots \oplus V_{s}$. The subspace $V_{1}$ defines a left-invariant distribution $H G \subset T G$ on $G$. We fix a left-invariant inner product on $H G$. An absolutely continuous curve $\gamma$ in $G$ whose velocity vector $\gamma^{\prime}(t)$ is contained in $H_{\gamma(t)} G$ for almost every $t$ is called a horizontal curve. Any horizontal curve has an associated length defined using the left-invariant inner product, i.e., by integrating the norm of its tangent vector. Since $V_{1}$ generates the whole Lie algebra, any two points of $G$ can be connected by horizontal curves. Let $p, q \in G$, the Carnot-Carathéodory metric between $p$ and $q$ is denoted by $d_{c}(p, q)$ and is defined as the infimum of length of horizontal curves that join $p$ and $q$. We call Carnot group the data of $G$, its stratification, the inner product on its first stratum, and consequently the distance $d_{c}$.

Since the inner product on $H G$ is left invariant, the Carnot metric on $G$ is left invariant as well. Different choices of inner product on $H G$ result in Carnot metrics that are biLipschitz equivalent. The Hausdorff dimension of $G$ with respect to a Carnot metric is given by $\sum_{i=1}^{s} i \cdot \operatorname{dim}\left(V_{i}\right)$.

Recall that, for a simply connected nilpotent Lie group $G$ with Lie algebra $\mathfrak{g}$, the exponential map exp $: \mathfrak{g} \rightarrow G$ is a diffeomorphism. Under this identification the Lesbegue measure on $\mathfrak{g}$ is a Haar measure on $G$. Furthermore, the exponential map induces a oneto-one correspondence between Lie subalgebras of $\mathfrak{g}$ and connected Lie subgroups of $G$.

Let $G$ be a Carnot group with Lie algebra $\mathfrak{g}=V_{1} \oplus \cdots \oplus V_{s}$. Since $\lambda_{t}: \mathfrak{g} \rightarrow \mathfrak{g}(t>0)$ is a Lie algebra automorphism and $G$ is simply connected, there is a unique Lie group automorphism $\Lambda_{t}: G \rightarrow G$ whose differential at the identity is $\lambda_{t}$. For each $t>0, \Lambda_{t}$ is a similarity with respect to the Carnot metric: $d\left(\Lambda_{t}(p), \Lambda_{t}(q)\right)=t d(p, q)$ for any two points $p, q \in G$. For a Lie group isomorphism $f: G \rightarrow G^{\prime}$ between two Carnot groups, the corresponding Lie algebra isomorphism $f_{*}: \operatorname{Lie}(G) \rightarrow \operatorname{Lie}\left(G^{\prime}\right)$ is strata preserving if and only if $f$ commutes with $\Lambda_{t}$ for all $t>0$; that is, if $f \circ \Lambda_{t}=\Lambda_{t} \circ f$.

\subsection{The Baker-Campbell-Hausdorff formula}

Let $G$ be a simply connected nilpotent Lie group with Lie algebra $\mathfrak{g}$. The exponential map $\exp : \mathfrak{g} \rightarrow G$ is a diffeomorphism. One can then pull back the group operation from $G$ to get a group structure on $\mathfrak{g}$. This group structure can be described by the Baker-CampbellHausdorff formula (BCH formula in short), which expresses the product $X * Y(X, Y \in \mathfrak{g})$ 
in terms of the iterated Lie brackets of $X$ and $Y$. The group operation in $G$ will be denoted by $\cdot$. The pull-back group operation $*$ on $\mathfrak{g}$ is defined as follows. For $X, Y \in \mathfrak{g}$, define

$$
X * Y=\exp ^{-1}(\exp X \cdot \exp Y)
$$

Then the first a few terms of the $\mathrm{BCH}$ formula ([CG], page 11) is given by:

$$
X * Y=X+Y+\frac{1}{2}[X, Y]+\frac{1}{12}[X,[X, Y]]-\frac{1}{12}[Y,[X, Y]]+\cdots .
$$

\subsection{Pansu differentiability theorem}

First the definition:

Definition 2.1. Let $G$ and $G^{\prime}$ be two Carnot groups endowed with Carnot metrics, and $U \subset G, U^{\prime} \subset G^{\prime}$ open subsets. A map $F: U \rightarrow U^{\prime}$ is Pansu differentiable at $x \in U$ if there exists a strata preserving homomorphism $L: G \rightarrow G^{\prime}$ such that

$$
\lim _{y \rightarrow x} \frac{d\left(F(x)^{-1} * F(y), L\left(x^{-1} * y\right)\right)}{d(x, y)}=0 .
$$

In this case, the strata preserving homomorphism $L: G \rightarrow G^{\prime}$ is called the Pansu differential of $F$ at $x$, and is denoted by $d F(x)$.

The following result (except the terminology) is due to Pansu $[\mathrm{P}]$.

Theorem 2.1. Let $G, G^{\prime}$ be Carnot groups, and $U \subset G, U^{\prime} \subset G^{\prime}$ open subsets. Let $F: U \rightarrow U^{\prime}$ be a quasiconformal map. Then $F$ is almost everywhere Pansu differentiable. Furthermore, at a.e. $x \in U$, the Pansu differential $d F(x): G \rightarrow G^{\prime}$ is a strata preserving isomorphism.

\section{Fiber-preserving quasisymmetric maps}

In this section we prove Theorem 1.1 .

Let $X, Y$ be $(\alpha, L)$-fibered quasimetric spaces and $F: X \rightarrow Y$ an $\eta$-quasisymmetric map that sends fibers of $X$ onto fibers of $Y$. We shall show that $F$ is a $(K, C)$-quasi-similarity, where $K$ depends only on $\eta, \alpha, L$ and the quasimetric constants of $X, Y$.

Lemma 3.1. If two fibers $U$ and $V$ in $X$ are parallel, then $F(U)$ and $F(V)$ are parallel fibers in $Y$.

Proof. Suppose $F(U)$ and $F(V)$ are not parallel. By Condition (1.5), after possibly switching $U$ and $V$, we may assume that there exist a sequence $x_{i} \in U$ such that $d\left(F\left(x_{i}\right), F(V)\right) \rightarrow$ $\infty$. Since $U$ and $V$ are parallel, for each $i$, there exists $v_{i} \in V$ such that $d\left(x_{i}, v_{i}\right)=d(U, V)$. Since by Condition (1.2) the set $U$ is path connected and unbounded, there are points on $U$ at arbitrary distance from any point. Hence, for each $i$, there exists $u_{i} \in U$ such that $d\left(x_{i}, u_{i}\right)=d\left(x_{i}, v_{i}\right)$ 
Since $d\left(F\left(x_{i}\right), F(V)\right) \rightarrow \infty$, we have $d\left(F\left(x_{i}\right), F\left(v_{i}\right)\right) \rightarrow \infty$. The quasisymmetry condition and the fact $d\left(x_{i}, u_{i}\right)=d\left(x_{i}, v_{i}\right)$ imply that $d\left(F\left(x_{i}\right), F\left(u_{i}\right)\right) \rightarrow \infty$.

Because of Condition (1.3), we can take a fiber $U^{\prime} \neq F(U)$ parallel to $F(U)$. Again by Condition (1.2) choose $y_{i} \in U^{\prime}$ so that $d\left(F\left(x_{i}\right), y_{i}\right)=d\left(F(U), U^{\prime}\right)$. Then $\frac{d\left(F\left(x_{i}\right), y_{i}\right)}{d\left(F\left(x_{i}\right), F\left(u_{i}\right)\right)} \rightarrow 0$ as $i \rightarrow \infty$. Now the quasisymmetry condition for $F^{-1}$ implies

$$
\frac{d\left(x_{i}, F^{-1}\left(y_{i}\right)\right)}{d(U, V)}=\frac{d\left(x_{i}, F^{-1}\left(y_{i}\right)\right)}{d\left(x_{i}, u_{i}\right)} \rightarrow 0 \text { as } i \rightarrow \infty .
$$

It follows that $d\left(x_{i}, F^{-1}\left(y_{i}\right)\right) \rightarrow 0$. Since $x_{i} \in U$ and $F^{-1}\left(y_{i}\right) \in F^{-1}\left(U^{\prime}\right)$, we have $d\left(U, F^{-1}\left(U^{\prime}\right)\right)=0$, contradicting Condition (1.4) and the fact $U^{\prime} \neq F(U)$.

The next two results are similar to Lemma 15.3 and Corollary 15.4 in [PW]. The proofs are modifications of their arguments.

Lemma 3.2. There exists $K_{1} \geq 1$ depending only on $\eta, \alpha$ and $L$ so that for any two parallel fibers $U_{1}, U_{2}$ in $X$ and any $p, q \in U_{1}$ satisfying $d(p, q) \geq L \cdot d\left(U_{1}, U_{2}\right)$ and $d(F(p), F(q)) \geq$ $L \cdot d\left(F\left(U_{1}\right), F\left(U_{2}\right)\right)$ we have

$$
\frac{1}{K_{1}} \cdot \frac{d\left(F\left(U_{1}\right), F\left(U_{2}\right)\right)}{d\left(U_{1}, U_{2}\right)} \leq \frac{d(F(p), F(q))}{d(p, q)} \leq K_{1} \cdot \frac{d\left(F\left(U_{1}\right), F\left(U_{2}\right)\right)}{d\left(U_{1}, U_{2}\right)} .
$$

Proof. By Condition (1.2), there exists a geodesic space $\left(\widetilde{X}_{1}, d_{1}\right)$ and an $L$-biLipschitz map $f_{1}:\left(U_{1}, d\right) \rightarrow\left(\widetilde{X}_{1}, d_{1}^{\alpha}\right)$. The assumption $d(p, q) \geq L \cdot d\left(U_{1}, U_{2}\right)$ implies

$$
d_{1}\left(f_{1}(p), f_{1}(q)\right) \geq\left(d\left(U_{1}, U_{2}\right)\right)^{1 / \alpha} .
$$

There is some integer $k \geq 2$ such that

$$
(k-1) \cdot\left(d\left(U_{1}, U_{2}\right)\right)^{1 / \alpha} \leq d_{1}\left(f_{1}(p), f_{1}(q)\right)<k \cdot\left(d\left(U_{1}, U_{2}\right)\right)^{1 / \alpha} .
$$

The biLipschitz property of $f_{1}$ implies

$$
\frac{1}{L}(k-1)^{\alpha} \cdot d\left(U_{1}, U_{2}\right) \leq d(p, q)<L k^{\alpha} \cdot d\left(U_{1}, U_{2}\right)
$$

Since $\left(\widetilde{X}_{1}, d_{1}\right)$ is a geodesic space, there are points $p=p_{0}, p_{1}, \ldots, p_{k}=q$ in $U_{1}$ such that $\frac{1}{2} d\left(U_{1}, U_{2}\right)^{\frac{1}{\alpha}} \leq d_{1}\left(f_{1}\left(p_{i}\right), f_{1}\left(p_{i+1}\right)\right) \leq d\left(U_{1}, U_{2}\right)^{\frac{1}{\alpha}}$. We have

$$
\frac{1}{L 2^{\alpha}} \cdot d\left(U_{1}, U_{2}\right) \leq d\left(p_{i}, p_{i+1}\right) \leq L \cdot d\left(U_{1}, U_{2}\right)
$$

Since $U_{1}$ and $U_{2}$ are parallel, Lemma 3.1 implies that $F\left(U_{1}\right)$ and $F\left(U_{2}\right)$ are also parallel. Let $q_{i} \in U_{2}$ be a point such that $d\left(F\left(p_{i}\right), F\left(q_{i}\right)\right)=d\left(F\left(U_{1}\right), F\left(U_{2}\right)\right)$. We have

$$
d\left(p_{i}, p_{i+1}\right) \leq L \cdot d\left(U_{1}, U_{2}\right) \leq L \cdot d\left(p_{i}, q_{i}\right) .
$$

Since $F$ is $\eta$-quasisymmetric, we have

$$
d\left(F\left(p_{i}\right), F\left(p_{i+1}\right)\right) \leq \eta(L) \cdot d\left(F\left(p_{i}\right), F\left(q_{i}\right)\right)=\eta(L) \cdot d\left(F\left(U_{1}\right), F\left(U_{2}\right)\right) .
$$


By Condition (1.2) again, there exists some geodesic space $\left(\widetilde{Y}_{1}, \rho_{1}\right)$ and an $L$-biLipschitz map $g_{1}:\left(F\left(U_{1}\right), d\right) \rightarrow\left(\widetilde{Y}_{1}, \rho_{1}^{\alpha}\right)$. It follows that

$$
\rho_{1}\left(g_{1} \circ F\left(p_{i}\right), g_{1} \circ F\left(p_{i+1}\right)\right) \leq(L \eta(L))^{\frac{1}{\alpha}} \cdot d\left(F\left(U_{1}\right), F\left(U_{2}\right)\right)^{\frac{1}{\alpha}} .
$$

The triangle inequality for the metric space $\left(\tilde{Y}_{1}, \rho_{1}\right)$ implies

$$
\rho_{1}\left(g_{1} \circ F(p), g_{1} \circ F(q)\right) \leq k(L \eta(L))^{\frac{1}{\alpha}} \cdot d\left(F\left(U_{1}\right), F\left(U_{2}\right)\right)^{\frac{1}{\alpha}} .
$$

Now the biLipschitz property of $g_{1}$ implies

$$
d(F(p), F(q)) \leq L \cdot \rho_{1}^{\alpha}\left(g_{1} \circ F(p), g_{1} \circ F(q)\right) \leq L^{2} \eta(L) k^{\alpha} \cdot d\left(F\left(U_{1}\right), F\left(U_{2}\right)\right) .
$$

Now the second inequality in (3.1) follows from (3.2) and (3.3). Finally, we notice that the second inequality for $F^{-1}$ is equivalent to the first inequality for $F$.

Lemma 3.3. The following holds for any two distinct parallel fibers $U_{1}, U_{2}$ and any two distinct $p, q \in U_{1}$ :

$$
\frac{1}{K_{1}^{3}} \cdot \frac{d\left(F\left(U_{1}\right), F\left(U_{2}\right)\right)}{d\left(U_{1}, U_{2}\right)} \leq \frac{d(F(p), F(q))}{d(p, q)} \leq K_{1}^{3} \cdot \frac{d\left(F\left(U_{1}\right), F\left(U_{2}\right)\right)}{d\left(U_{1}, U_{2}\right)},
$$

where $K_{1}$ is the constant in Lemma 3.2.

Proof. Let $U_{1}$ and $U_{2}$ be two distinct parallel fibers, and $p, q \in U_{1}$ be distinct. Pick two points $p_{0}, q_{0} \in U_{1}$ that satisfies $d\left(p_{0}, q_{0}\right)>L \cdot d\left(U_{1}, U_{2}\right)$ and $d\left(F\left(p_{0}\right), F\left(q_{0}\right)\right)>L$. $d\left(F\left(U_{1}\right), F\left(U_{2}\right)\right)$. By Lemma 3.2 we have

$$
\frac{1}{K_{1}} \cdot \frac{d\left(F\left(U_{1}\right), F\left(U_{2}\right)\right)}{d\left(U_{1}, U_{2}\right)} \leq \frac{d\left(F\left(p_{0}\right), F\left(q_{0}\right)\right)}{d\left(p_{0}, q_{0}\right)} \leq K_{1} \cdot \frac{d\left(F\left(U_{1}\right), F\left(U_{2}\right)\right)}{d\left(U_{1}, U_{2}\right)} .
$$

By Condition (1.3) there exist a sequence of fibers $U_{\lambda_{i}} \neq U_{1}$ parallel to $U_{1}$ such that $U_{\lambda_{i}}$ converges to $U_{1}$. For sufficiently large $i$, we have

$$
\min \left\{d(p, q), d\left(p_{0}, q_{0}\right)\right\}>L \cdot d\left(U_{1}, U_{\lambda_{i}}\right)
$$

and

$$
\min \left\{d(F(p), F(q)), d\left(F\left(p_{0}\right), F\left(q_{0}\right)\right)\right\}>L \cdot d\left(F\left(U_{1}\right), F\left(U_{\lambda_{i}}\right)\right) .
$$

Now Lemma 3.2 applied to $U_{1}, U_{\lambda_{i}}$ and $p, q$ yields

$$
\frac{1}{K_{1}} \cdot \frac{d\left(F\left(U_{1}\right), F\left(U_{\lambda_{i}}\right)\right)}{d\left(U_{1}, U_{\lambda_{i}}\right)} \leq \frac{d(F(p), F(q))}{d(p, q)} \leq K_{1} \cdot \frac{d\left(F\left(U_{1}\right), F\left(U_{\lambda_{i}}\right)\right)}{\left.d\left(U_{1}, U_{\lambda_{i}}\right)\right)} .
$$

Similarly, we have

$$
\frac{1}{K_{1}} \cdot \frac{d\left(F\left(U_{1}\right), F\left(U_{\lambda_{i}}\right)\right)}{d\left(U_{1}, U_{\lambda_{i}}\right)} \leq \frac{d\left(F\left(p_{0}\right), F\left(q_{0}\right)\right)}{d\left(p_{0}, q_{0}\right)} \leq K_{1} \cdot \frac{d\left(F\left(U_{1}\right), F\left(U_{\lambda_{i}}\right)\right)}{\left.d\left(U_{1}, U_{\lambda_{i}}\right)\right)} .
$$

It follows that

$$
\frac{1}{K_{1}^{2}} \cdot \frac{d\left(F\left(p_{0}\right), F\left(q_{0}\right)\right)}{d\left(p_{0}, q_{0}\right)} \leq \frac{d(F(p), F(q))}{d(p, q)} \leq K_{1}^{2} \cdot \frac{d\left(F\left(p_{0}\right), F\left(q_{0}\right)\right)}{d\left(p_{0}, q_{0}\right)} .
$$

Now (3.4) follows from (3.5) and (3.6). 
Lemma 3.3 says that for any fiber $U$ of $X$, the restriction $\left.F\right|_{U}$ is a $\left(K_{1}^{3}, C\right)$-quasisimilarity for some constant $C>0$ that may depend on the fiber $U$. Next lemma states that this constant $C$ can be chosen to be independent of the fiber.

Lemma 3.4. There is some constant $C>0$ such that $\left.F\right|_{U}$ is a $\left(K_{2}, C\right)$-quasi-similarity for any fiber $U$ of $X$, where $K_{2}$ is a constant depending only on $\eta, \alpha, L$ and the quasimetric constants of $X, Y$.

Proof. Fix a fiber $U_{0}$ (in $X$ ) and let $U$ be an arbitrary fiber in $X$. By Lemma 3.3, there are constants $C_{0}, C>0$ such that $\left.F\right|_{U_{0}}$ is a $\left(K_{1}^{3}, C_{0}\right)$-quasi-similarity and $\left.F\right|_{U}$ is a $\left(K_{1}^{3}, C\right)$ quasi-similarity. It suffices to show that there is a constant $D$ depending only on $\eta, \alpha, L$ and the quasimetric constants of $X, Y$ such that $C_{0} / D \leq C \leq D C_{0}$.

Let $M \geq 1$ be a quasimetric constant for both $X$ and $Y$. Fix $x \in U$ and $x_{0} \in U_{0}$, and pick $y \in U$ and $y_{0} \in U_{0}$ such that

$$
d\left(x_{0}, y_{0}\right)=d(x, y)=10 M \cdot d\left(x, x_{0}\right)
$$

The generalized triangle inequality in $X$ applied to $x, x_{0}, y_{0}$ implies

$$
\frac{1}{2 M} \cdot d(x, y)=\frac{1}{2 M} \cdot d\left(x_{0}, y_{0}\right) \leq d\left(x, y_{0}\right) \leq 2 M \cdot d\left(x_{0}, y_{0}\right)=2 M \cdot d(x, y)
$$

The quasisymmetry condition now implies

$$
d\left(F\left(x_{0}\right), F\left(y_{0}\right)\right) / \eta(2 M) \leq d\left(F(x), F\left(y_{0}\right)\right) \leq \eta(2 M) \cdot d\left(F\left(x_{0}\right), F\left(y_{0}\right)\right)
$$

and

$$
d(F(x), F(y)) / \eta(2 M) \leq d\left(F(x), F\left(y_{0}\right)\right) \leq \eta(2 M) \cdot d(F(x), F(y)) .
$$

It follows that

$$
\frac{1}{(\eta(2 M))^{2}} \cdot d\left(F\left(x_{0}\right), F\left(y_{0}\right)\right) \leq d(F(x), F(y)) \leq(\eta(2 M))^{2} \cdot d\left(F\left(x_{0}\right), F\left(y_{0}\right)\right) .
$$

This together with the quasi-similarity property of $\left.F\right|_{U_{0}}$ and $\left.F\right|_{U}$ implies $C_{0} / D \leq C \leq D C_{0}$, where $D=(\eta(2 M))^{2} K_{1}^{6}$.

Lemma 3.5. $F$ is a $(K, C)$-quasi-similarity, where $K$ depends only on $\eta, \alpha, L$ and the quasimetric constants of $X, Y$. .

Proof. Let $p, q \in X$ be arbitrary. Let $U, V$ be fibers in $X$ such that $p \in U, q \in V$ (we may have $U=V)$. Pick $x \in U$ such that $d(p, x)=d(p, q)$. Then

$$
d(F(p), F(q)) \leq \eta(1) \cdot d(F(p), F(x)) \leq \eta(1) K_{2} C \cdot d(p, x)=\eta(1) K_{2} C \cdot d(p, q) .
$$

So we have the upper bound for $d(F(p), F(q))$. The same argument applied to $F^{-1}$ yields the lower bound for $d(F(p), F(q))$. 
The proof of Theorem 1.1 is now complete.

\section{An Example}

Recall that two closed subsets $U$ and $V$ of a quasimetric space are defined to be parallel if $d(u, V)=d(v, U)$ for any $u \in U, v \in V$. The following example shows that the conclusion of Theorem 1.1 fails if we replace "parallel" with the weaker condition $H D(U, V)<\infty$.

Let $X=Y=\mathbb{C}$ be the complex plane with the usual metric. It is well known that for any $\alpha>-1$, the map $f_{\alpha}: \mathbb{C} \rightarrow \mathbb{C}$ given by $f_{\alpha}(z)=|z|^{\alpha} z$ is a quasisymmetric map. Let $\alpha>-1, \alpha \neq 0$, define a map $F_{\alpha}: \mathbb{C} \rightarrow \mathbb{C}$ as follows:

$$
F_{\alpha}(z)=\left\{\begin{aligned}
f_{\alpha}(z) & \text { if }|z| \leq 1 \\
z & \text { if }|z| \geq 1
\end{aligned}\right.
$$

Then it is clear that $F_{\alpha}$ is a quasiconformal map, and hence is quasisymmetric. The fibers in $X$ are horizontal lines, and the fibers in $Y$ are images of horizontal lines under $F_{\alpha}$. A direct calculation shows that all fibers of $Y$ are 10-biLipschitz to the real line. So Condition (1.2) is satisfied by both $X$ and $Y$. All the conditions in Definition 1.1 are satisfied, provided we replace "parallel" with the weaker condition $H D(U, V)<\infty$. However, $F_{\alpha}$ is not biLipschitz due to the distorsion around the origin.

\section{Applications}

\subsection{Quasisymmetric maps on Carnot groups with reducible first stratum}

In this section we use Theorem 1.1 to prove Theorem 1.2, Specifically, we show that every quasisymmetric map on Carnot groups with reducible first stratum is biLipschitz.

Let $G$ be a Carnot group with Lie algebra $\mathfrak{g}=V_{1} \oplus \cdots \oplus V_{s}$. Assume there is a nontrivial proper linear subspace $W_{1} \subset V_{1}$ that is invariant under the action of the group of strata-preserving automorphisms of $\mathfrak{g}$. Let $\mathfrak{h}$ be the subalgebra of $\mathfrak{g}$ generated by $W_{1}$. We denote the connected Lie subgroup of $G$ with Lie algebra $\mathfrak{h}$ by $H$ and refer to it as the subgroup generated by $W_{1}$. Notice that $H$ is also a Carnot group and its lie algebra can be written as $\mathfrak{h}=W_{1} \oplus \cdots \oplus W_{s}$. In general, there is some integer $1 \leq \bar{s} \leq s$ such that $W_{\bar{s}} \neq 0$ and $W_{j}=0$ for $j>\bar{s}$. Let $\langle\cdot, \cdot\rangle$ be an inner product on $V_{1}$ and $d$ the left-invariant sub-Riemannian Carnot metric on $G$ determined by $\langle\cdot, \cdot\rangle$.

We recall the following:

Proposition 4.1. (Proposition 3.4, XX2]) Let $G$ and $G^{\prime}$ be two Carnot groups. Let $W_{1}$ and $W_{1}^{\prime}$ be two subspaces of the first strata of the stratifications of $\operatorname{Lie}(G)$ and $\operatorname{Lie}\left(G^{\prime}\right)$, respectively. Let $H$ and $H^{\prime}$ be the groups generated by $W_{1}$ and $W_{1}^{\prime}$, respectively. Let $F$ : $G \rightarrow G^{\prime}$ be a quasisymmetric map. If $d F(x)\left(W_{1}\right) \subset W_{1}^{\prime}$ for a.e. $x \in G$, then $F$ sends each left coset $U$ of $H$ into a left coset of $H^{\prime}$.

Now let $F: G \rightarrow G$ be an $\eta$-quasisymmetric map. By Pansu's differentiability theorem, at almost everywhere $x \in G$ the map $F$ is Pansu differentiable and the Pansu differential 
$d F(x): \mathfrak{g} \rightarrow \mathfrak{g}$ is a strata-preserving automorphism. The assumption on $W_{1}$ in Theorem 1.2 implies that $d F(x)\left(W_{1}\right)=W_{1}$. Now Proposition 4.1 implies that $F$ sends left cosets of $H$ to left cosets of $H$, where $H$ is the subgroup generated by $W_{1}$.

Theorem 1.2 shall follow from Theorem 1.1 once we verify the conditions in Definition 1.1. Here the fibers are left cosets of $H$. Since $H$ is also a Carnot group, $(H, d)$ is a geodesic space. So Condition (1.2) is satisfied for $\alpha=L=1$. Conditions (1.3), (1.4) and (1.5) will be verified in Lemmas 4.2, 4.3 and 4.4, respectively.

Given a Lie algebra $\mathfrak{n}$ and a subalgebra $\mathfrak{s} \subset \mathfrak{n}$, the normalizer of $\mathfrak{s}$ in $\mathfrak{n}$ is defined by:

$$
\mathcal{N}_{\mathfrak{n}}(\mathfrak{s})=\{X \in \mathfrak{n}:[X, \mathfrak{s}] \subset \mathfrak{s}\}
$$

It is easy to see that $\mathcal{N}_{\mathfrak{n}}(\mathfrak{s})$ is a Lie subalgebra of $\mathfrak{n}$ and $\mathfrak{s}$ is an ideal in $\mathcal{N}_{\mathfrak{n}}(\mathfrak{s})$. Let $N$ be a connected Lie group with Lie algebra $\mathfrak{n}$. Let $S$ be the Lie subgroup of $N$ with Lie algebra s. Let $d$ be a left invariant distance on $N$. Lemmas 4.2 and 4.3 are valid for all connected and simply connected nilpotent Lie groups $N$ with a left invariant distance. In particular, they hold true for the Carnot group $G$ and left cosets of $H$.

Lemma 4.2. For any proper Lie subalgebra $\mathfrak{s}$ of a nilpotent Lie algebra $\mathfrak{n}$, we have

$$
\mathcal{N}_{\mathfrak{n}}(\mathfrak{s}) \neq \mathfrak{s} .
$$

Consequently, for any left coset $U$ of $S$, there exist a sequence of left cosets $U_{i}$ of $S$ that are parallel to $U$ and converge to $U$.

Proof. For the first claim we induct on the degree of nilpotency. The claim holds when $\mathfrak{n}$ is abelian since in this case $\mathcal{N}_{\mathfrak{n}}(\mathfrak{s})=\mathfrak{n}$. Suppose the claim holds for all $k$-step nilpotent Lie algebras. Let $\mathfrak{n}$ be $(k+1)$-step. We assume $\mathcal{N}_{\mathfrak{n}}(\mathfrak{s})=\mathfrak{s}$ and will derive a contradiction from this. Notice the center $C(\mathfrak{n})$ of $\mathfrak{n}$ lies in $\mathcal{N}_{\mathfrak{n}}(\mathfrak{s})=\mathfrak{s}$. So we have a proper Lie subalgebra $\mathfrak{s} / C(\mathfrak{n})$ of the $k$-step nilpotent Lie algebra $\mathfrak{n} / C(\mathfrak{n})$. By the induction hypothesis, we have $\mathcal{N}_{\mathfrak{n} / C(\mathfrak{n})}(\mathfrak{s} / C(\mathfrak{n})) \neq \mathfrak{s} / C(\mathfrak{n})$. Let $\pi: \mathfrak{n} \rightarrow \mathfrak{n} / C(\mathfrak{n})$ be the natural projection. It is easy to check that $\pi^{-1}\left(\mathcal{N}_{\mathfrak{n} / C(\mathfrak{n})}(\mathfrak{s} / C(\mathfrak{n}))\right)=\mathcal{N}_{\mathfrak{n}}(\mathfrak{s})$. Since $\pi^{-1}(\mathfrak{s} / C(\mathfrak{n}))=\mathfrak{s}$, we obtain $\mathcal{N}_{\mathfrak{n}}(\mathfrak{s}) \neq \mathfrak{s}$, contradicting the assumption $\mathcal{N}_{\mathfrak{n}}(\mathfrak{s})=\mathfrak{s}$.

Regarding the second part of the lemma, let $K$ be the connected subgroup of $N$ with Lie algebra $\mathcal{N}_{\mathfrak{n}}(\mathfrak{s})$. Since $\mathfrak{s}$ is an ideal in $\mathcal{N}_{\mathfrak{n}}(\mathfrak{s})$, we see that $S$ is a normal subgroup of $K$. From (4.1) we deduce that $K$ contains $S$ properly. There exist $k_{i} \in K \backslash S$ such that $k_{i} \rightarrow e$. So for any left coset $U=g S$, the left cosets $U_{i}=g k_{i} S$ converge to $U$. Finally we claim that $U_{i}$ is parallel to $U$. Indeed, for any $p=g s_{0} \in U$,

$$
d\left(p, U_{i}\right)=d\left(g s_{0}, g k_{i} S\right)=d\left(e, s_{0}^{-1} k_{i} S\right)=d\left(e, k_{i} \cdot k_{i}^{-1} s_{0}^{-1} k_{i} S\right)=d\left(e, k_{i} S\right)
$$

and for any $q=g k_{i} s_{0} \in U_{i}$,

$$
\begin{aligned}
d(q, U) & =d\left(g k_{i} s_{0}, g S\right) \\
& =d\left(S k_{i} s_{0}, e\right)=d\left(S k_{i} s_{0} k_{i}^{-1} \cdot k_{i}, e\right)=d\left(S k_{i}, e\right)=d\left(e, S k_{i}\right)=d\left(e, k_{i} S\right),
\end{aligned}
$$

where in the last equality we used the fact that $S k_{i}=k_{i} S$. 
Notice that the last part of the preceding proof shows that for any $g \in N$ and any $k \in K$, the two left cosets $g S$ and $g k S$ are parallel.

Next lemma says that two different left costs of $S$ can never get arbitrarily close.

Lemma 4.3. If $U$ and $V$ are two distinct left cosets of $S$, then $d(U, V)>0$.

Proof. Set $\mathfrak{s}_{0}=\mathfrak{s}$ and define inductively $\mathfrak{s}_{j}=\mathcal{N}_{\mathfrak{n}}\left(\mathfrak{s}_{j-1}\right)$. From (4.1) we have that $\mathfrak{s}_{j}$ properly contains $\mathfrak{s}_{j-1}$ unless $\mathfrak{s}_{j-1}=\mathfrak{n}$. Since $\mathfrak{n}$ is finite dimensional, there is some $k$ such that $\mathfrak{s}_{k-1} \neq \mathfrak{n}$ and $\mathfrak{s}_{k}=\mathfrak{n}$. Let $S_{j}$ be the connected Lie subgroup of $N$ with Lie algebra $\mathfrak{s}_{j}$. Then $S_{j-1}$ is a proper normal sugroup of $S_{j}$ for $j \leq k$. It follows that for any $g \in S_{j} \backslash S_{j-1}$, the two left cosets $S_{j-1}$ and $g S_{j-1}$ are parallel. Hence $d\left(S_{j-1}, g S_{j-1}\right)>0$.

Now let $U$ and $V$ be two distinct left cosets of $S$. After applying a left translation we may assume $U=S$ and $V=g S$ for some $g \notin S$. There is some $j \leq k$ such that $g \in S_{j} \backslash S_{j-1}$. It follows that

$$
d(U, V)=d(S, g S) \geq d\left(S_{j-1}, g S_{j-1}\right)>0
$$

since $S \subset S_{j-1}$.

For the proof of the next lemma we will work under the assumption of Theorem 1.2,

Let $\langle\cdot, \cdot\rangle$ be an inner product on $\mathfrak{g}=V_{1} \oplus \cdots \oplus V_{s}$ such that $V_{i}$ and $V_{j}$ are perpendicular to each other for $i \neq j$. Define a "quasi norm" on $\mathfrak{g}$ as follows.

$$
\left\|\sum_{i} x_{i}\right\|=\sum_{i}\left\langle x_{i}, x_{i}\right\rangle^{\frac{1}{2 i}}, \text { where } x_{i} \in V_{i} .
$$

Next define $\bar{d}(x, y)=\|(-x) * y\|$ for $x, y \in \mathfrak{g}$. It is well known that every Carnot metric $d$ on $\mathfrak{g}$ is biLipschitz equivalent to $\bar{d}$ : there exists some constant $C_{0} \geq 1$ such that

$$
\frac{1}{C_{0}} \cdot \bar{d}(x, y) \leq d(x, y) \leq C_{0} \cdot \bar{d}(x, y)
$$

for any $x, y \in \mathfrak{g}$. Here we identified $G$ and $\mathfrak{g}$ via the exponential map.

Lemma 4.4. If $U$ and $V$ are two left cosets of $H$ that are not parallel, then $H D(U, V)=\infty$.

Proof. Suppose $U$ and $V$ are not parallel and $H D(U, V)<\infty$. We shall obtain a contradiction. After applying a left translation we may assume $U=H$ and $V=g H$ for some $g \in G \backslash H$. The last part of the proof of Lemma 4.2 shows that for any $k$ in the normalizer $K$ of $H$ the two fibers $H$ and $k H=H k$ are parallel. So $g \notin K$. Below we will identify $\mathfrak{g}$ with $G$ via the exponential map and do calculations in the Lie algebra using BCH formula. So we let $Y \notin \mathcal{N}_{\mathfrak{g}}(\mathfrak{h})$ be such that $H D(\mathfrak{h}, Y * \mathfrak{h})<\infty$. Since

$$
H D(Y * \mathfrak{h}, Y * \mathfrak{h} *(-Y)) \leq d(e,-Y)
$$

we see that $H D(\mathfrak{h}, Y * \mathfrak{h} *(-Y)) \leq C$ for some constant $C>0$.

We write $Y=\sum_{i=1}^{s} Y_{i}$ with $Y_{i} \in V_{i}$ and let $j \geq 1$ be the index such that $Y_{j} \notin \mathcal{N}_{\mathfrak{g}}(\mathfrak{h})$ and $Y_{i} \in \mathcal{N}_{\mathfrak{g}}(\mathfrak{h})$ for all $i<j$. By replacing $Y$ with $Y *\left(-Y_{1}-\ldots-Y_{j-1}\right)$, we may assume $Y_{i}=0$ for $i<j$. Since $\left[Y_{j}, \mathfrak{h}\right] \not \subset \mathfrak{h}$ and $\mathfrak{h}$ is generated by the first stratum $W_{1}$, there is some 
$X \in W_{1}$ such that $\left[Y_{j}, X\right] \notin \mathfrak{h}$. Notice that $\left[Y_{j}, X\right] \in V_{j+1}$. Since $H D(\mathfrak{h}, Y * \mathfrak{h} *(-Y)) \leq C$, for any $t \in \mathbb{R}$, there is some $X^{\prime} \in \mathfrak{h}\left(X^{\prime}\right.$ may depend on $\left.t\right)$ such that

$$
d\left(\left(-X^{\prime}\right) * Y *(t X) *(-Y), 0\right)=d\left(Y *(t X) *(-Y), X^{\prime}\right) \leq C .
$$

We next calculate $Z:=Y *(t X) *(-Y)$ and $A:=\left(-X^{\prime}\right) * Z$. We have

$$
Z=e^{\operatorname{ad} Y}(t X)=t\left\{X+[Y, X]+\cdots+\frac{1}{k !}(\operatorname{ad} Y)^{k}(X)+\cdots+\frac{1}{(s-1) !}(\operatorname{ad} Y)^{s-1}(X)\right\},
$$

where $\operatorname{ad} Y: \mathfrak{g} \rightarrow \mathfrak{g}$ is the linear map given by ad $Y(B)=[Y, B]$ for $B \in \mathfrak{g}$. Write $Z=Z_{1}+\cdots+Z_{s}$ with $Z_{i} \in V_{i}$. Since $Y_{i}=0$ for $i<j$, we have

$$
\frac{1}{k !}(\operatorname{ad} Y)^{k}(X) \in V_{j+2} \oplus \cdots \oplus V_{s} \text { for all } k \geq 2 .
$$

So the terms $Z_{i}$ with $i \leq j+1$ is determined by $t(X+[Y, X])$. Since $X \in V_{1}$ we have $Z_{1}=t X, Z_{i}=0$ for $2 \leq i \leq j$ and $Z_{j+1}=t\left[Y_{j}, X\right]$.

Write $A=A_{1}+\cdots+A_{s}$ with $A_{i} \in V_{i}$. Notice that $\left[-X^{\prime}, Z\right]$ and all the iterated brackets in the $\mathrm{BCH}$ formula for $\left(-X^{\prime}\right) * Z$ are the sum of an element of $\mathfrak{h}$ and an element in $V_{j+2} \oplus \cdots \oplus V_{s}$. So $A_{j+1}$ is completely determined by $-X^{\prime}+Z$. It follows that $A_{j+1}$ is the sum of an element of $W_{j+1}$ and $t\left[Y_{j}, X\right]$ (recall $\mathfrak{h}=W_{1} \oplus \cdots \oplus W_{s}$ ). Write $\left[Y_{j}, X\right]=B+B^{\perp}$, where $B \in W_{j+1}$ and $B^{\perp} \in V_{j+1}$ is perpendicular to $W_{j+1}$ with respect to the inner product $<,>$ on $\mathfrak{g}$. Since $\left[Y_{j}, X\right] \notin \mathfrak{h}$, we have $B^{\perp} \neq 0$. Hence $A_{j+1}$ equals the sum of $t B^{\perp}$ and an element of $W_{j+1}$. It follows that

$$
d\left(\left(-X^{\prime}\right) * Y *(t X) *(-Y), 0\right) \geq \frac{1}{C_{0}} \cdot\left\|\left(-X^{\prime}\right) * Y *(t X) *(-Y)\right\| \geq \frac{1}{C_{0}} \cdot\left|t B^{\perp}\right|^{\frac{1}{j+1}} \rightarrow \infty,
$$

as $t \rightarrow \infty$ since $B^{\perp} \neq 0$. This contradicts (4.2).

We have verified all the conditions in Definition 1.1 and the proof of Theorem 1.2 is now complete.

Remark 4.3. Lemma 4.4 is equivalent to the following statement: if the Hausdorff distance between $H$ and $g \mathrm{Hg}^{-1}$ is finite, then $g$ lies in the normalizer of $H$. We believe this is true for any left invariant distance on any connected, simply connected nilpotent Lie group $N$ and any proper Lie subgroup $H$.

\subsection{Other applications}

In this subsection we give a couple of other applications of Theorem 1.1. The first is to quasiconformal maps of Heisenberg groups that send vertical lines to vertical lines. The second is to quasisymmetric maps of ideal boundary of certain amenable hyperbolic locally compact groups.

We first show that quasiconformal maps of the Heisenebrg groups $H^{n}=\mathbb{R}^{2 n} \times \mathbb{R}$ that permute vertical lines are biLipschitz. These maps are lifts of those biLipschitz maps of $\mathbb{R}^{2 n}$ that preserve the standard sympletic form on $\mathbb{R}^{2 n}$. The reader is referrd to $[\mathrm{T}]$ and $[\mathrm{BHT}]$ for more details. 
Proposition 4.5. Let $F: H^{n} \rightarrow H^{n}$ be a quasiconformal map of the Heisenberg group. If $F$ maps vertical lines to vertical lines, then $F$ is biLipschitz.

Proof. Recall that by [HK, Theorem 4.7], $F$ is quasisymmetric. The fibers are the vertical lines. There is some $L \geq 1$ such that the vertical lines are $L$-biLipschitz to $\left(\mathbb{R},|\cdot|^{\frac{1}{2}}\right)$, where $|\cdot|$ is the usual metric on $\mathbb{R}$. So Condition (1.2) in Definition 1.1 is satisfied for $\alpha=1 / 2$ and $L$. It is easy to see that the other three conditions in Definition 1.1 are also satisfied. By Theorem 1.1, $F$ is biLipschitz.

We next give a simple proof of Dymarz's theorem.

Theorem 4.6 ([D, Theorem 1]). Let $N$ be a Carnot group equipped with a left invariant Carnot metric $d$ and $\mathbb{Q}_{m}(m \geq 2)$ the $m$-adics with standard metric $d_{m}$. Then every quasisymmetric map $N \times \mathbb{Q}_{m} \rightarrow N \times \mathbb{Q}_{m}$ is biLipschitz. Here the metric on $N \times \mathbb{Q}_{m}$ is given by $d^{\prime}\left(\left(x_{1}, y_{1}\right),\left(x_{2}, y_{2}\right)\right)=\max \left\{d\left(x_{1}, x_{2}\right), d_{m}\left(y_{1}, y_{2}\right)\right\}$.

Recall that the standard metric $d_{m}$ on $\mathbb{Q}_{m}$ is given by:

$$
d_{m}\left(\sum a_{i} m^{i}, \sum b_{i} m^{i}\right)=m^{-(k+1)},
$$

where $k$ is the smallest index for which $a_{i} \neq b_{i}$.

Proof. The fibers of $N \times \mathbb{Q}_{m}$ are the subsets $N \times\{y\}\left(y \in \mathbb{Q}_{m}\right)$. Notice that $\left(\mathbb{Q}_{m}, d_{m}\right)$ is perfect and totally disconnected. So the fibers of $N \times \mathbb{Q}_{m}$ are exactly the connected components of $N \times \mathbb{Q}_{m}$. Hence every quasisymmetric map $F$ of $N \times \mathbb{Q}_{m}$ permutes the fibers. Each fiber is isometric to a Carnot group and so is geodesic. Hence Condition (1.2) is satisfied for $\alpha=L=1$. The perfectness of $\mathbb{Q}_{m}$ implies that parallel fibers are not isolated (Condition (1.3)). Distance between distinct fibers is clearly positive (Condition (1.4)). All fibers are parallel, so Condition (1.5) is vacuous. By Theorem 1.1 every quasisymmetric map of $N \times \mathbb{Q}_{m}$ is biLipschitz.

\section{References}

[BHT] Z. Balogh, R. Hoefer-Isenegger, J. Tyson, Lifts of Lipschitz maps and horizontal fractals in the Heisenberg group, Ergodic Theory Dynam. Systems 26 (2006), no. 3, 621-651.

[CG] L. Corwin, F. Greenleaf, Representations of nilpotent Lie groups and their applications, Part I. Basic theory and examples, Cambridge Studies in Advanced Mathematics, 18. Cambridge University Press, Cambridge, 1990.

[CO] M. Cowling, A. Ottazzi, Global contact and quasiconformal mappings of Carnot groups, http://arxiv.org/pdf/1408.1778.pdf

[CR] M. Cowling, M. Reimann, Quasiconformal mappings on Carnot groups: three examples, Harmonic analysis at Mount Holyoke (South Hadley, MA, 2001), 2003, pp. $111-118$. 
[D] T. Dymarz, Quasisymmetric maps of boundaries of amenable hyperbolic groups, Indiana University Mathematics Journal 63 (2014), 329-343.

[HK] J. Heinonen, P. Koskela, Quasiconformal maps in metric spaces with controlled geometry, Acta Math. 181 (1998), no. 1, 1-61.

[P] P. Pansu, Metriques de Carnot-Caratheodory et quasiisometries des espaces symetriques de rang un, Ann. of Math. (2) 129 (1989), no. 1, 1-60.

[Pi] M. C. Piaggio, Orlicz spaces and the large scale geometry of Heintze groups, http://arxiv.org/pdf/1411.6381.pdf

[PW] P. Pankka, J. Wu, Geometry and Quasisymmetic Parametrization of Semmes Spaces, to appear in Revista Mat. Iberoamericana.

[SX] N. Shanmugalingam, X. Xie, A Rigidity Property of Some Negatively Curved Solvable Lie Groups, Comment. Math. Helv. 87 (2012), no. 4, 805-823.

[T] P. Tang, Regularity and extremality of quasiconformal homeomorphisms on CR 3manifolds, Ann. Acad. Sci. Fenn. Math. 21 (1996), no. 2, 289-308.

[V] J. Väisälä, The free quasiworld. Freely quasiconformal and related maps in Banach spaces, Quasiconformal geometry and dynamics, Banach Center Publ. (Lublin) 48 (1996), 55-118.

[X1] X. Xie, Large scale geometry of negatively curved $\mathbb{R}^{n} \rtimes \mathbb{R}$, Geom. Topol. 18 (2014), no. $2,831-872$.

[X2] X. Xie, Quasisymmetric maps on reducible Carnot groups, Pacific Journal of Mathematics 265 (2013), no. 1, 113-122.

[X3] X. Xie, Some examples of quasiisometries of nilpotent Lie groups, to appear in Journal fur die reine und angewandte Mathematik.

[X4] X. Xie, Quasiisometries of negatively curved homogeneous manifolds associated with Heisenberg groups, to appear in Journal of Topology.

[X5] X. Xie, Quasiconformal maps on non-rigid Carnot groups, see http://front.math.ucdavis.edu/1308.3031

Addresses:

Enrico Le Donne: Department of Mathematics and Statistics, P.O. Box 35 (MaD), FI-40014 University of Jyvaskyla, Finland. E-mail: ledonne@msri.org

Xiangdong Xie: Dept. of Mathematics and Statistics, Bowling Green State University, Bowling Green, OH, U.S.A. E-mail: xiex@bgsu.edu 